him was, not that he did not support the doctrine, but that nowhere did he attempt to sort out all the wealth of detail collected, and classify it according to the specialised functions. My attempt to do so in regard to the female genital functions is the first, but I am sure it will not be the last, attempt of the sort.

In conclusion, I would like myself to state how cordially I agree with much that has been said and written about many wild generalisations that have been made in papers which give no evidence of first-hand knowledge; and also to point out that the pure physiologist will not go far in the solution of the problems connected with the internal secretions until he takes more heed of the clinical and pathological aspects of the subject.

I am, Sir, yours faithfully,

Liverpool, Feb. 2nd, 1914.

W. BI.AIR BELL.

\section{DOSAGE AND THE METRIC SYSTEM.}

To the Editor of THE LANCET.

SIR,-Your readers interested in the application of the metric system to medicine and pharmacy will note with much satisfaction Mr. G. E. M. Johnson's announcement in your last issue (p. 357) that the Decimal Association has been assured by the General Medical Council of the adoption of the metric system in the dosage of the forthcoming edition of the British Pharmacopcia. Possibly this announcement gives to my proposal in your issue of Jan. 24th (p.231) a somewhat belated appearance. Yet, as qualifying this criticism, I may plead that the proposal was originally advanced in 1903, ${ }^{1}$ and that, in so far as I knew, it had attracted no attention. In these circumstances a ten years' interval may perhaps excuse its repetition.

I am, Sir, yours faithfully,

Harley-street, W., Feb. 2nd, $1914 . \quad$ C. O. HAWTHORNE.

\section{GOATS' MILK.}

To the Editor of THE LANCET.

SIR,-The correspondence about milk ought not to close without a word about goat's milk.

Goats practically never have tubercle, therefore their milk can be given without pasteurising; further, it can be obtained fresh and clean. I once caught in a test-tube milk from the teat of a goat being milked by a boy; the test-tube was placed in an incubator and remained sterile. With respect to cost you will find an estimate in the British Goat Society's Monthly Circular; it figures out for the keep only of the goats at $1 \% 4$. per quart of milk, but goat's milk is not an article of commerce, and it is necessary for the child's father to take some trouble in the matter, which, if the object is explained to him and he lives in the country, he will be willing to do. Groats are small and cleanly animals, and can be kept in health even in the centre of a large town; their milk is said to be better for infants than cow's milk because the curd is finer.

I am, Sir, yours faithfully,

Deronshire.place, W., Jan. 31st, 1914. WALTER EDMUNDS.

*** We have been compelled by pressure on our correspondence columns to hold further letters on the subject of milk sterilisation over until next week. - ED. L.

1 Polyclinic Journal, April, 1903, p. 138

\section{TREATMENT OF GASTRIC AND DUODENAL} PERFORATION.

\author{
To the Editor of THE LANCET.
}

SIR,-The tamponade method of Mr. E. M. Corner is one that most surgeons have been obliged to content themselves with occasionally in desperate cases and when the perforation is found to be in. accessible to ready suture. That it can be a means of saving life as a substitute for laborious suturing there can be no doubt, and Mr. Corner is to be congratulated on his effort to bring the method prominently before surgeons.

In the discussion on this subject I did not notice any mention of a valuable auxiliary to any method of dealing with abdominal catastrophes of this class-that is, cases of acute peritoneal sepsis. I refer to the injection of camphor and olive oil into the cavity. I have been using this for about 18 months, and have formed the opinion that it is of real value; in fact, as regards treatment alone I am inclined to place it along. side of the Murphy proctoclysis and the Fowler position. The procedure is simple and rapid; for an adult 100 grammes of a 1 per cent. solution of camphor in olive oil is poured into the peritoneal cavity as soon as the perforation is found and secured by whatever method is deemed suitable, the cavity having been previously cleansed by mopping out, not flushing. The method is equally effective and equally useful in cases of fulminating appendicitis and such-like causes of diffuse septic peritonitis. A point of practical im. portance is that if silkworm gut sutures are used for the abdominal and suprapubic drainage wounds, they may either be placed before the oil is used or a pair of cotton gloves should be put over the rubber ones as the oiled silkworm gut is slippery to handle.

The theoretical proposition is that the camphor acts quickly as a cardiac stimulant, and that the oil is taken up rapidly by the lymphatics and tem. porarily blocks them, thus diminishing the absorption of toxins. If oil has any value in preventing the formation of adhesions, then this is an additional incentive to use the method. As there appears to be some difficulty in procuring the mixture ready sterilised, I may say that Messrs. Burroughs and Wellcome have prepared for me flasks in two sizes, 50 grammes for children and 100 grammes for adults. For those who desire a full account of this treatment $I$ would refer them to an excellent article in Lyon Chirurgical, December, 1910-January, 1911, by M. Vignard and M. Arnaud. I am, Sir, yours faithfully, Newport, Mon., Jan. 28tb, 1914. W. J. GREER.

\section{ASEPTIC URETERAL CATHETERISATION. To the Eator of THE LANCET.}

SIR,-In ThE LANCET of Jan. 24th Mr. R. J. Willan describes his ingenious appliance for per. forming aseptic ureteral catheterisation. The same ideal can be obtained by means of a far simpler appliance which $\mathbf{I}$ adopted some time ago. It consists of a tube made of fine linen or calico. open at both ends, about half an inch in diameter and slightly longer than the ureteric catheter. The tube is sterilised by boiling, and the end of the previously sterilised catheter is inserted by meaus of sterile forceps in the end of the tube. The cathetel is then worked along the tube exactly as a honsewife works a bodkin along a seam. As soon as 